\title{
Análise Retrospectiva das Neoplasias de Ânus em Pacientes Atendidos no HC-FMRP-USP de 1979 a 2004 e Revisão da Literatura
}

\author{
Retrospective Analysis of Patients with Anal Tumors Diagnosed at the School of \\ Medicine of Ribeirão Preto Hospital and Clinics (HC-FMRP), Between 1979 \\ and 2004 and Literature Review
}

\author{
ROGÉRIO SERAFIM PARRA ${ }^{1}$; ANDREZA REGINADE BRITO ${ }^{1}$; JOSÉ JOAQUIM RIBEIRO DAROCHA²; \\ OMAR FÉRES ${ }^{2}$ \\ ${ }^{1}$ Pós-graduando junto à Área de Clínica Cirúrgica do Depto. de Cirurgia e Anatomia da FMRP-USP; \\ ${ }^{2}$ Professor Doutor junto à Disciplina de Coloproctologia do Depto. de Cirurgia e Anatomia da FMRP-USP.
}

PARRA RS; BRITO AR; ROCHA JJR; FÉRES O. Análise Retrospectiva das Neoplasias de Ânus em Pacientes Atendidos no HC-FMRPUSP de 1979 a 2004 e Revisão de Literatura. Rev bras Coloproct, 2007;27(2): 119-129.

RESUMO: Foram analisados, retrospectivamente, 49 casos de pacientes com neoplasia de ânus, sendo 23 de $1979-1996$ e 26 de 1997-2004, 49\% masculinos e 51\% femininos. Em 81,6\% dos pacientes o tratamento foi adjuvante, com radioterapia e esquema de NIGRO, 12,2\% metástases à distância e 36,7\% perderam o seguimento. Óbitos ocorreram em 20,4\%. Notou-se diminuição da necessidade da cirurgia de Miles (30,8\% vs 43,3\%). A perda de seguimento foi menor (19,5\% vs 56,5\%), mas a mortalidade foi discretamente maior $(23,1 \%$ vs $\mathbf{1 7}, 4 \%)$. O tratamento neo-adjuvante com radio e quimioterapia em $96 \%$ dos pacientes com neoplasia anal foi capaz de promover remissão da lesão na maioria dos casos $(57,5 \%)$, confirmados pela biópsia da cicatriz residual, evitando-se amputação cirúrgica do reto. Dados mais recentes mostram que $45 \%$ dos pacientes permanecem sem recidiva (seguimento médio 3,5 anos). A alta taxa de mortalidade e o diagnóstico de lesões avançadas podem decorrer da procura tardia do serviço médico.

Palavras chave: neoplasia maligna de ânus, incidência, epidemiologia.

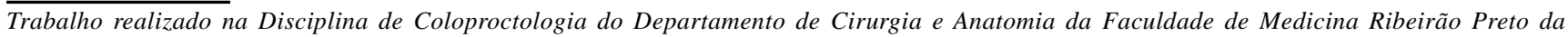
Universidade de São Paulo. Departamento de Cirurgia e Anatomia do Hospital das Clínicas da Faculdade de Medicina Ribeirão Preto da Universidade de São Paulo (HCFMRP-USP).

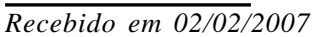

Aceito para publicação em 12/06/2007 


\section{INTRODUÇÃO}

Uma variedade de neoplasias pode afetar o canal anal e o ânus. Eles constituem achado raro na prática médica ${ }^{12,18,24,48}$, representando cerca de $1-2 \%$ dos tumores em coloproctologia e cerca de $4 \%$ dos tumores malignos anorretais ${ }^{8,27,32,40}$. Entretanto, o cirurgião, seguramente, irá encontrar alguns pacientes com esta patologia durante sua carreira ${ }^{18}$, o que reforça a importância do assunto.

De todos os cânceres do ânus, $85 \%$ são do canal anal ${ }^{1}$. O tipo histológico mais freqüente, representando cerca de $75 \%$ das neoplasias da região anal, é o carcinoma epidermóide 1, 5, 7, 10, 14, 18, 19, 22, 48. Entre outros, estão incluídos o adenocarcinoma (geralmente invasão secundária de um adenocarcinoma de reto baixo que desce para o canal anal), o carcinoma de células basalóides, o linfoma, o melanoma e o tumor de Busch-Loweistein.

Os tumores do canal anal são mais comuns em mulheres e em homens homossexuais ${ }^{2-6,11,29}$. Podem ocorrem em qualquer faixa etária, mas são mais comuns na sexta e sétima décadas de vida ${ }^{2,6,30}$. Os principais fatores de risco incluem: Sexo feminino ${ }^{2-5}$ intercurso sexual anal e promiscuidade ${ }^{3,5,14,29}$, tabagismo $^{5,14,29}$, raça negra ${ }^{3,12}$, infecção pelo HPV e ou HIV/SIDA ${ }^{3,5}, 11,13,14,17,29$, idade avançada ${ }^{2}$. Estudo da Suécia mostra evidências de que doenças benignas, tais como, doença hemorroidária ${ }^{5,29}$ e inflamação anal crônica (por exemplo, Doença de Crohn) ${ }^{5}$, aumentam o risco de carcinoma espinocelular anal.

Frisch et al. ${ }^{5}$ demonstraram que comportamento sexual está relacionado ao aumento de risco de Carcinoma espinocelular (CEC) anal, havendo aumento comprovado entre homens homossexuais, principalmente se promíscuos, e entre heterossexuais promíscuos. Comprovou-se que, em pessoas com tal comportamento, existe aumento na taxa de infecção HPV, e conseqüentemente, aumento na taxa de CEC anal. A taxa da doença também é maior entre fumantes (único fator não relacionado ao sexo). Não existem evidencias de que a imunossupressão secundária à infecção pelo HIV, em seus primeiros dois anos, aumente a taxa de CEC anal. No entanto, com a introdução dos anti retrovirais a partir de 1996 e o conseqüente aumento na sobrevida dos pacientes com SIDA houve aumento na taxa de CEC anal, provavelmente secundária ao aumento no tempo de imunossupressão ao qual tais pacientes são expostos ${ }^{5,21,35}$.
A incidência do câncer anal aumentou nos 30 anos últimos anos, tanto em homens como em mulheres ${ }^{2-5,12,15}$. Este aumento se deve, principalmente, ao surgimento da epidemia pelo HIV/SIDA e à mudança de comportamento sexual da população ${ }^{11,17,21,35,36}$. A infecção pelo HIV e a co-infecção por HPV (condiloma perianal) foram identificadas como fator de risco independente para carcinoma anal, sendo que o risco de carcinoma invasivo em pacientes infectado pelo HPV é aumentado pelo HIV e pela co-infecção anal pelo $\mathrm{HPV}^{13}$.

Sintomas perianais são comuns na prática clínica e condições anorretais benignas, tais como hemorróidas, fístulas crônicas, radioterapia prévia podem ter características clínicas similares ao câncer de canal anal. Para evitar atrasos diagnósticos, os médicos devem ser capazes de reconhecer os casos suspeitos de serem neoplasia, já que a terapia clínica pode evitar a necessidade de operações mutilantes, tais como a amputação abdômino perineal do reto e do ânus (RAP), com colostomia definitiva e perda de todo aparelho esfincteriano ${ }^{20}$.

Os sintomas principais do câncer anal incluem sangramento retal, prurido anal, secreção mucosa, tenesmo, sensação de protuberância ou nodulação anal, alteração do hábito intestinal. Até mesmo as metástases nodais inguinais podem surgir como sintoma primário, antes que o tumor primário acarrete sintomas perianais significativos ${ }^{51}$.

No momento do diagnóstico inicial cerca de 53\% dos pacientes têm a doença localizada. $38 \%$ dos pacientes se apresentam com doença regional ${ }^{2}$. As metástases à distância no momento do diagnóstico variam de $10 \%$ a $25 \%$ 2, 6, 23, 24,48. A região inguinal é o sitio mais comum de metástases das neoplasias da região anal. A presença de acometimento ganglionar, que ocorre em cerca de 15 a $20 \%$ dos pacientes ${ }^{48}$, é um importante fator prognóstico. A presença de metástases nodais inguinais é um fator prognóstico independente para recidiva local ou persistência da doença após terapia clínica combinada e importante para a mortalidade global de pacientes com neoplasia da região anal ${ }^{24}$. A adenomegalia inguinal, após tratamento clínico com radio e quimioterapia ou após tratamento cirúrgico com RAP, foi o sinal mais freqüente de recidiva da doença ${ }^{41}$.

É muito importante que a localização anatômica e o tipo histológico das neoplasias da região anal sejam definidos no momento do diagnóstico, já que o tratamento inicial pode ser diferente. A definição anatômica 
para o câncer de canal anal versus câncer de margem anal é baseada na relação do tumor com a borda anal ${ }^{21}$. O tratamento inicial para as neoplasias de margem anal é a terapia cirúrgica ${ }^{18}{ }^{19}$. Já para o carcinoma espinocelular (CEC) de canal anal a terapia primária padrão ouro é a terapia combinada que consiste em radioterapia em conjunto com quimioterapia ${ }^{1,6,8,10,16,18-}$ 20,22,27,32,38, resultando em bom controle local da doença a, assim, evitando a RAP, aumentando a sobrevida geral, livre da doença e evitando a necessidade de colostomia definitiva ${ }^{6}$.

Na década de 1970-1980, até o estudo pioneiro de Nigro e colaboradores, a RAP do reto a ânus era a terapia padrão para o CEC anal. A terapia combinada, descrita primeiramente por Nigro e colaboradores em $1974^{50}$ consiste na administração de Mitomicina (10 a $15 \mathrm{mg} / \mathrm{m}^{2}$, bolus intravenoso no dia 1), com fluoruracil (infusão intravenosa por $4-5$ dias, 750 a $1000 \mathrm{mg} / \mathrm{m}^{2}$ ). A radioterapia é aplicada durante 3 a 7 semanas, começando, seja no primeiro dia, ou imediatamente após o ciclo de quimioterapia (dia 6). A dose de radiação varia de 30 a $50 \mathrm{~Gy}$, sendo que a maioria dos centros utiliza campos paralelos. O campo de radiação pode ser ajustado para incluir os linfonodos inguinais acometidos pela doença, caso necessário.

Entre 4 a 6 semanas após término do esquema de Nigro, todos os pacientes são examinados sob anestesia para avaliar se houve controle local (confirmado pela biópsia da cicatriz residual pós terapia combinada) ou persistência da doença, o que indica RAP.

Subseqüentemente dois grandes estudos randomizados da EORTC (European Organization for Research and Treatment of Cancer) e da UKCCCR (United Kingdom Coordinating Committee Oncology Group $)^{63,64}$ demonstraram a superioridade do uso concomitante da 5FU a mitomicina associada à radioterapia sobre a radioterapia sozinha ou terapia cirúrgica.

Diversos trabalhos demonstram resposta completa à terapia combinada ${ }^{50}$, com desaparecimento da neoplasia sem necessidade de RAP. A resposta ao esquema varia de $80 \%{ }^{6}, 90 \%$ a até $100 \%{ }^{19}$, confirmada pela biópsia da cicatriz residual após o tratamento clínico inicial. A maioria dos estudos mostra que a terapia combinada promove controle local significativamente melhor se comparada com pacientes que recebem somente radioterapia. Mesmo para grandes tumores, maiores que $5 \mathrm{~cm}$, que apresentam menor resposta à terapia combinada (cerca de 50 a 75\%), devemos, inicialmente, tentar a terapia combinada, proporcionando ao paciente uma chance de cura, com boa sobrevida, sem a necessidade de colostomia definitiva ${ }^{19}$. Entretanto, os tumores em estágio avançado, com doença nodal inguinal e grande tamanho tumoral $(>5 \mathrm{~cm})$ apresentaram queda significativa no controle local da doença em 5 anos e diminuição na taxa de sobrevida ${ }^{37,39}$.

Entretanto, alguns poucos trabalhos, tais como Tobulou e colaboradores ${ }^{7}$, afirmam que a radioterapia isolada desde 1980 é a terapia de escolha (com intenção curativa) para os casos de CEC de canal anal na França. A terapia concomitante com quimioterapia seria reservada e indicada como a melhor forma de tratamento para tumores localmente avançados, ou sejam, que sejam maiores que $4 \mathrm{~cm}$ ou que apresentem comprometimento nodal.

Chauvenic et al ${ }^{30}$ afirmam que a idade foi o principal determinante se o paciente seria submetido ou não a quimioterapia, associada à radioterapia. Embora afirme que a terapia combinada seja considerada padrão ouro no tratamento do câncer de canal anal, apenas 55\% dos pacientes com tumores T3/T4 neste trabalho foram submetidos à terapia combinada e os demais à radioterapia isolada, devido principalmente à idade avançada de tais pacientes. A alta taxa de complicações tardias observadas nos pacientes que receberam terapia combinada, neste estudo, fornece evidências de que tal tratamento combinado deve ser reservado a pacientes mais jovens sem co-morbidades. Serkies e cols citam que a terapia combinada está associada a uma alta taxa de toxicidade (cerca de 54\%, com efeitos hematológicos e gastrintestinais) ${ }^{39}$.

Como diversos estudos demonstraram que a terapia combinada provoca toxicidade bem documentada, na tentativa de buscar alternativas à toxicidade da mitomicina, Hung e cols ${ }^{43}$ aplicaram um esquema alternativo, substituindo a mitomicina pela cisplatina em 92 pacientes. A terapia combinada, utilizando a cisplatina ao invés de mitomicina, foi bem tolerada, sem a toxicidade apresentada pela última, podendo ser usado com altas taxas de controle local, sobrevida geral e preservação do esfíncter, e sobrevida livre de doença.

Nem todos os autores confirmam o achado de toxicidade com a terapia combinada descrita por Nigro. Park e cols ${ }^{41}$ submeteram 23 pacientes a terapia combinada associada (quimioterapia associada à radioterapia externa) e os mesmos não apresentaram sérios efeitos colaterais e complicações tardias.

O intervalo livre de doença em 4 anos (sem recorrência) é de cerca de $70 \%$, e nos pacientes com 
tumores T3/T4 foi de $78 \%$ com a terapia combinada e $60 \%$ com radioterapia isolada ${ }^{41}$.

Outro estudo francês ${ }^{33}$ mostrou excelentes resultados utilizando radioterapia isolada em pacientes com tumores T1/T2. No entanto, a terapia combinada proposta por Nigro deve ser preferida em tumores localmente avançados, maiores ou iguais a $2 \mathrm{~cm}$ ou com doença nodal, a fim de melhorar a sobrevida livre de colostomia, mantendo o aparelho esfincteriano anal e, conseqüentemente, permitindo continência ao paciente ${ }^{8,34}$.

A cirurgia radical proposta por Miles, em 1908, consiste na amputação abdômino perineal do reto e ânus (RAP) e fica reservada para recorrências tumorais após o tratamento clínico inicial após terapia, ou para os casos em que resposta ao tratamento foi insuficiente $1,8,16,27,38,48$. Tais casos, geralmente, são de tumores localmente avançados $\left(\mathrm{N}^{+}\right.$ou $\left.\mathrm{T} \geq 4 \mathrm{~cm}\right)$. A descrição cirúrgica pode ser encontrada em diversos livros textos ${ }^{50}$ ou em sua forma original, descrita por Miles ${ }^{51}$.

ARAP, após a recidiva ou recorrência tumoral, pode proporcionar sobrevida longa em pacientes selecionados com câncer de canal anal. No entanto, oferece pouca esperança para os pacientes com tumores $\mathrm{T} 4$ ou envolvimento nodal N2/N3 ${ }^{28}$. A RAP pode ainda ser utilizada em pacientes que apresentem incontinência fecal pré-operatória ${ }^{47}$.

Apesar do aumento significativo na incidência da doença, nos últimos 30 anos, diversos estudos mostram melhora significativa na sobrevida nos pacientes com neoplasia maligna da região anal ${ }^{2,6,12,41}$. No geral, em 5 anos, a sobrevida média gira em torno de 70 a $80 \%{ }^{8,41}$. Já para aqueles pacientes submetidos à terapia de resgate (RAP) após falha terapêutica (recidiva ou recorrência) a sobrevida média em 5 anos gira em torno de $50 \%{ }^{10}$. Cerca de $20 \%$ dos pacientes que apresentam metástases à distância no momento do diagnóstico permanecem vivos dentro de cinco anos de acompanhamento, comparados com $80 \%$ dos pacientes que apresentam doença localizada ${ }^{12}$.

Os principais fatores relacionados à diminuição da sobrevida nas neoplasias da região anal incluem tumores maiores que $5 \mathrm{~cm}$, acometimento de órgãos adjacentes durante o tratamento cirúrgico de salvação após falha à terapia combinada inicial, envolvimento nodal após a terapia combinada e margens positivas ${ }^{16}$. O prognóstico depende do tamanho do tumor primário e da presença de disseminação nodal ${ }^{32}$. Os principais fatores de diminuição na sobrevida e de recorrência da doença são a doença persistente após terapia inicial com a terapia combinada, e doença nodal notada durante a cirurgia de salvação. A cirurgia de salvação após falha na terapia combinada é uma tentativa razoável para cura. Fatores independentes para melhor prognóstico incluem ausência de doença nodal ao salvamento, margens negativas e cirurgia de salvação potencialmente curativa após recorrência ou persistência da doença depois da terapia inicial com a terapia combinada ${ }^{16}$.

\section{OBJETIVO}

Avaliar o perfil desta afecção com relação à epidemiologia, tipo histológico, tratamento e sobrevida em 2 períodos de tempo diferentes, procurando analisar os dados durante todo o período e verificar se existem evidências de diferenças entre os pacientes com diagnóstico mais antigo com pacientes com diagnóstico mais recente: $1^{\circ}$ período: entre os anos de 1979 a 1996; $2^{\circ}$ período: entre os anos de 1997 a 2004.

\section{CASUÍSTICA E MÉTODO}

Foi realizada uma avaliação retrospectiva dos prontuários médicos de pacientes portadores das neoplasias da região anal, atendidos no Hospital das Clínicas da Faculdade de Medicina de Ribeirão Preto/ SP da Universidade de São Paulo (HCFMRP-USP) entre os anos de 1979 a 2004. Este período (1979-2004) foi subdividido em dois períodos: $1^{\circ}$ período (1979-1996) e $2^{\circ}$ período (1997-2004): $1^{\circ}$ período (1979 a 1996): inclui 23 pacientes; $2^{\circ}$ período (1997 a 2004): dados mais recentes, incluindo 27 pacientes.

No total são 50 pacientes e foram, confrontados os achados e diagnósticos entre os diferentes tipos de neoplasia em ambos os períodos, analisando dados referentes a: história clínica, exames subsidiários, resposta ao tratamento, cirurgias e evolução clínica.

Os dados foram distribuídos em tabelas para melhor compreensão e entendimento. Foram distribuídas as médias referentes ao período total (1979-2004) e as médias dos $1^{\circ}$ e $2^{\circ}$ períodos.

Resposta completa à terapia combinada foi definida como desaparecimento clínico do tumor, confirmado pela biópsia excisional da cicatriz cirúrgica e resultado negativo para neoplasia no anátomo-patológico.

O seguimento dos pacientes englobou os períodos de 1979 a 2004, ou então até a perda de seguimento dos doentes, ou até o óbito dos mesmos. 


\section{RESULTADOS}

Foram levantados 50 prontuários médicos de pacientes com neoplasia de canal anal, sendo 23 pacientes no período de $1979-1996$ ( $1^{\circ}$ período $)$ e 27 pacientes no período de 1997-2004 ( $2^{\circ}$ período).

A análise de todos os dados dos 50 pacientes, englobando os dois períodos (1979-2004), mostrou discreta predominância do sexo feminino ( $51 \%$ versus $49 \%$ ). Aidade média foi de 56,3 anos ${ }^{25,87}$. Os sintomas mais freqüentes foram sangramento (57\%), dor anal (53\%), presença de massa na região anal (47\%). Entre as patologias associadas temos imunossupressão (SIDA, terapia com corticoesteróides, pós-transplante renal) (12,3\%), condiloma perianal ${ }^{3,12}$, abscesso anal $(10,2 \%)$ e pacientes sabidamente HIV positivos ao diagnóstico (7\%) (Tabela 1).
O resultado da biópsia (Anátomo-patológico) mostrou $61,2 \%$ de Carcinoma de células escamosas, 20,4\% de Adenocarcinoma, 10,2\% de Carcinoma de células basalóides e $8,2 \%$ de outros resultados. A cirurgia foi realizada em $90 \%$ casos, sendo que $37 \%$ foram submetidos à amputação abdômino perineal do reto e ânus (RAP, Cirurgia de Miles) e 58\% submetidos à ressecção local da lesão residual ou cicatriz após terapia combinada. $81,6 \%$ dos pacientes tiveram tratamento adjuvante com o esquema proposto por Nigro e cols. (Tabela 2).

No total, $12,2 \%$ dos pacientes apresentavam metástases à distância no momento do diagnóstico; $36,7 \%$ perderam o seguimento durante o tempo de observação. Os óbitos foram confirmados em 20,4\% (Tabela 3).

Tabela 1 - Distribuição dos dados referentes à idade, sexo, sintomas e patologias associadas dos 50 pacientes (prontuários médicos) analisados retrospectivamente no HCFMRP-USP, no período de 1979 a 2004.

\begin{tabular}{lllll}
\hline Total de pacientes & Idade média & Sexo & Sintomas principais & Patologias associadas \\
\hline \multirow{2}{*}{50} & & & Imunossupressão: $12 \%$ \\
& & & \\
& 56,3 anos & $51 \%$ feminino & Sangramento: $57 \%$ & Condiloma perianal: $12 \%$ \\
& $25-87$ anos & $49 \%$ masculino & Dor anal : $53 \%$ & Abscesso anal: $10 \%$ \\
& & & Massa anal: $47 \%$ & HIV positivo: $7 \%$ \\
\hline
\end{tabular}

Tabela 2 - Distribuição dos dados referentes ao resultado do Anátomo-patológico, procedimento cirúrgico, resposta ao tratamento dos 50 pacientes analisados retrospectivamente no HCFMRP-USP, no período de 1979 a 2004.

\begin{tabular}{llll}
\hline Total de pacientes & Anátomo-patológico & Terapia combinada & $\begin{array}{l}\text { Cirurgia } \\
\text { (90\% dos pacientes) }\end{array}$ \\
\hline 50 & CEC 62\% & RAP 37\% & Biópsia cicatriz 58\% \\
& Adenocarcinoma 20\% & RT + QT 82\% & $\begin{array}{l}\text { Resposta completa ao } \\
\text { tratamento 60\% }\end{array}$ \\
& Cel. Basalóides 10\% & $(5 \mathrm{FU}+$ mitomicina+RT 50Gy) & \\
& & & \\
\hline
\end{tabular}

Tabela 3 - Distribuição dos dados referentes à presença de metástases à distância ao diagnóstico, perda de seguimento e taxa de mortalidade dos 50 pacientes analisados retrospectivamente no HCFMRP-USP, no período de 1979 a 2004.

\begin{tabular}{lccc}
\hline Total de pacientes & $\begin{array}{c}\text { Metástases à distância no } \\
\text { momento do diagnóstico }\end{array}$ & Perda de seguimento & Mortalidade \\
\hline $\mathrm{n}=50$ & $12 \%$ & $37 \%$ & $21 \%$ \\
\hline
\end{tabular}


Comparando-se os períodos 1979-1996 e 1997-2004 notamos que, no $1^{\circ}$ período, houve aumento no número proporcional de mulheres com a neoplasia de canal anal (54\% vs $48 \%$ ), aumento da idade média (61,4 anos versus 50,6 anos), sangramento como sintoma mais freqüente (vs dor), manutenção do Carcinoma Espinocelular como principal neoplasia $57,7 \%$, porém com aumento no número de casos de Adenocarcinoma (27\% vs $13 \%$ ) e Carcinoma de Células Basalóides (15\% vs 8,7\%). Nota-se também diminuição da necessidade RAP (Cirurgia de Miles) $(30,8 \%$ vs $43,3 \%)$ no $1^{\circ}$ período, perda de seguimento menor $(19,5 \%$ vs $56,5 \%)$, e mortalidade levemente maior $(23,1 \%$ vs $17,4 \%)$ (Tabela 4,5 e 6$)$.

\section{DISCUSSÃO}

As neoplasias da região anal assumem grande importância na era do HIV/AIDS. Diversos trabalhos mostram a associação de ambas patologias. Este trabalho visou uma análise epidemiológica das neoplasias da região anal no HCFMRP-USP, comparando os períodos de 1979-1996, quando a infecção pelo HIV não havia atingido níveis epidêmicos, com o período de 1997-

Tabela 4 - Distribuição comparativa dos dados (idade, sexo, sintomas, patologias associadas) entre os períodos de 1979-1996 e 1997-2004.

\begin{tabular}{lll}
\hline & $\mathbf{1}^{\mathbf{o}}$ período (1979-1996) & $\mathbf{2}^{\mathbf{0}}$ período (1997-2004) \\
\hline Total de pacientes & $\mathrm{n}=23$ & $\mathrm{n}=27$ \\
Idade média & 50,6 anos & 60,9 anos \\
Sexo & Masculino 52\% & Masculino 48\% \\
Sintomas principais & Feminino 48\% & Feminino 52\% \\
& Dor anal: $69,5 \%$ & Sangramento: $65,5 \%$ \\
& & Massa anal: $44,4 \%$ \\
& Massa: $52,2 \%$ & Mudança hábito Intestinal: $43 \%$ \\
Antecedentes ou & Sangramento: $47,8 \%$ & Dor anal: $38,5 \%$ \\
patologias associadas & Abscesso: $13 \%$ & Perda de peso: $31 \%$ \\
& Imunossupressão: $18,5 \%$ & \\
& Condiloma $9 \%$ & \\
& HIV: $9 \%$ & Trat. como hemorróidas antes do diagnóstico: $12 \%$ \\
& Condiloma: $18,5 \%$ & HIV: $11,5 \%$ \\
& & Abscesso: $7,7 \%$ \\
\hline
\end{tabular}

Tabela 5 - Distribuição comparativa dos dados (Anátomo-patológico, metástases à distância ao diagnóstico, utilização do esquema de Nigro, com radio e quimioterapia combinados e resposta completa ao esquema combinado) entre os períodos de 1979-1996 e 1997-2004.

\begin{tabular}{|c|c|c|}
\hline & 1979-1996, $1^{\circ}$ período & 1997-2004, $2^{\circ}$ período \\
\hline Tipo histológico & CEC: $65 \%$ & \\
\hline (Anátomo-patológico) & Adenocarcinoma: $13 \%$ & CEC: $59 \%$ \\
\hline Outros: $13,3 \%$ & Adenocarcinoma: $27 \%$ & \\
\hline Carcinoma Basalóide: $8,7 \%$ & Basalóide: $15 \%$ & \\
\hline Metástases à distância ao diagnóstico & $13 \%$ & $12 \%$ \\
\hline Terapia combinada & $65 \%$ & $95 \%$ \\
\hline Resposta completa à terapia combinada. & $58 \%$ & \\
\hline
\end{tabular}


Tabela 6 - Distribuição comparativa dos dados (realização da RAP, biópsia excisional da cicatriz residual após terapia combinada, presença de metástase nodal inguinal, perda de seguimento e média de seguimento dos pacientes) entre os períodos de 1979-1996 e 1997-2004.

\begin{tabular}{|c|c|c|}
\hline & 1979-1996, $1^{\circ}$ período & 1997-2004, $2^{\circ}$ período \\
\hline RAP & $43,3 \%$ & $34,2 \%$ \\
\hline Biópsia da cicatriz residual & & $58 \%$ \\
\hline Perda de seguimento & $56 \%$ & $19 \%$ \\
\hline Taxa de recidiva & & Após RAP: $63 \%$ \\
\hline & & Após exérese de cicatriz: $13 \%$ \\
\hline Média de seguimento & & 3,5 anos \\
\hline Metástases ganglionares & & $22 \%$ \\
\hline
\end{tabular}

2004, após estabelecimento da epidemia HIV/SIDA. Visou também verificar e analisar as diferenças entre os dados em ambos os períodos, ou seja, modificações na epidemiologia, apresentação diagnóstica, tratamento e prognóstico nos últimos anos, procurando encontrar outros fatores que possam alterar a incidência e ocorrência da doença em nosso serviço.

Diversos trabalhos mostram aumento significativo na incidência das neoplasias da região anal, tanto em homens como em mulheres ${ }^{2-5,12,15}$. Nosso trabalho confirma os dados de literatura. No $1^{\circ}$ período (1979-1996, 17 anos de estudo) foram catalogados 23 casos, enquanto no $2^{\circ}$ período (1997-2004, 7 anos de estudo) foram registrados 27 casos, praticamente $60 \%$ de aumento no número de casos da doença diagnosticados em nosso serviço.

As neoplasias do canal anal podem ocorrer em qualquer faixa etária, mas são mais comuns na sexta e sétima décadas de vida ${ }^{2,6,30}$. Em nossa estatística do $2^{\circ}$ período, a idade média foi de 61 anos, confirmando achados da literatura. Os tumores do canal anal são mais comuns em mulheres e em homens homossexuais ${ }^{2-5,6,11,29}$. Nossos dados mostram que não houve diferença significativa quanto ao sexo em ambos os períodos, o que confronta os dados da literatura. No entanto, os dados mais recentes ( $2^{\circ}$ período, de 1997 a 2004) mostram evidências de aumento (não significativo) no número de mulheres com neoplasia da região anal ( $52 \%$ versus $48 \%)$.

Os principais fatores de risco para as neoplasias da região anal incluem o sexo feminino ${ }^{2-5}$, intercurso sexual anal e promiscuidade ${ }^{3,5,14,29}$, tabagismo ${ }^{5,14,29}$, raça negra ${ }^{3,12}$, infecção pelo HPV e ou HIV/SIDA ${ }^{3,5}$, $11,13,14,17,29$, idade avançada ${ }^{2}$. Até mesmo algumas do- enças benignas, tais como doença hemorroidária ${ }^{5,29} \mathrm{e}$ inflamação anal crônica (por exemplo, Doença de Crohn $)^{5}$, aumentam o risco de carcinoma espinocelular anal. Em nosso trabalho as principais patologias associadas foram tabagismo, imunossupressão, HIV, condilomatose perianal (HPV).

A análise global dos dados deste trabalho mostrou que cerca de $10 \%$ dos pacientes portadores de neoplasia da região anal são portadores do vírus da Imunodeficiência Humana (HIV) (contra $12 \%$ no $2^{\circ}$ período) e que cerca de $15 \%$ dos pacientes possuíam evidências da infecção pelo Papiloma vírus (HPV), pela presença de condilomatose perianal e de canal anal (contra cerca de $19 \%$ no $2^{\circ}$ período). A imunossupressão, não apenas secundária à infecção pelo HIV e conseqüentemente Síndrome da Imunodeficiência Adquirida (SIDA), está associada ao aparecimento do câncer anal, já que cerca de $20 \%$ dos pacientes apresentavam evidências de imunossupressão, seja pela infecção pelo HIV, seja por uso crônico de corticóides, ou então imunossupressão secundária a medicações após transplante renal.

Os sintomas principais do câncer anal incluem sangramento retal, prurido anal, secreção mucosa, tenesmo, sensação de protuberância ou nodulação anal (massa anal), alteração do hábito intestinal, perda de peso. Até mesmo as metástases nodais inguinais podem surgir como sintoma primário, antes que o tumor primário acarrete sintomas perianais significativos ${ }^{51}$. Em nosso trabalho, os principais sintomas associados foram sangramento (2/3), massa anal (1/2), alteração no hábito intestinal (43\%), dor anal (40\%) e perda de peso $(31 \%)$. 
A literatura mostra que $85 \%$ de todos os cânceres do ânus são do canal anal ${ }^{1}$. O tipo histológico mais frequiente é o carcinoma epidermóide (CEC) ${ }^{1,5,7}$, 10, 14, 18, 19, 22, 48. Entre outros, estão incluídos o adenocarcinoma (geralmente invasão secundária de um adenocarcinoma de reto baixo que desce para o canal anal), o carcinoma de células basalóides, o linfoma, o melanoma e o tumor de Busch-Loweistein. Em nosso trabalho, o CEC se mantém como a neoplasia mais freqüente em ambos os períodos, representando $62 \%$ dos tumores da região anal. Nota-se, no entanto, aumento importante nos casos de adenocarcinomas, refletindo o aumento nos casos de neoplasias de reto baixo com invasão do canal anal, nos últimos anos.

Diversos trabalhos relatam que no momento do diagnóstico cerca de $53 \%$ dos pacientes têm a doença localizada e $38 \%$ dos pacientes se apresentam com doença regional ${ }^{2}$. Em nossa estatística cerca de 2/3 dos pacientes apresentavam-se com doença localizada. As metástases a distância no momento do diagnóstico, importante fator prognóstico, variam de $10 \%$ a $25 \%^{2,6,23,24,48}$. A região inguinal é o sítio mais comum de metástases das neoplasias da região anal ${ }^{24}$. Em nosso trabalho, encontramos $22 \%$ de acometimento nodal inguinal, o que bate com os dados de literatura. A presença de metástases nodais inguinais é um fator prognóstico independente para recidiva local ou persistência da doença após terapia clínica combinada e importante para a mortalidade global de pacientes com neoplasia da região anal $^{24}$.

Desde o trabalho de Nigro e cols, dois grandes trials randomizados (UKCCCR e ECRTC) confirmaram os excelentes resultados do uso concomitante da radioterapia externa associada à $5 \mathrm{FU}$ e mitomicina, oferecendo resultados superiores se comparados com radioterapia sozinha ou tratamento cirúrgico isolado (RAP). A resposta completa à terapia combinada chega a $80 \%$. Nossos dados mostram que, no período de 1979 a 1996, a terapia combinada era utilizada com menor freqüência (cerca de $2 / 3$ dos pacientes) e, desta forma, havia maior necessidade de RAP (44\%). Os dados mais recentes de 1997 a 2004 mostram que 95\% dos pacientes utilizaram a terapia combinada (o único paciente que não fez o esquema não tinha condições clínicas, por tem insuficiência renal e hepática em estágios avançados). Desta maneira, a necessidade de RAP diminuiu para $34 \%$.

A resposta completa ao esquema de Nigro, confirmada pela biópsia da cicatriz residual e resultado negativo no anátomo-patológico, foi de $60 \%$. A terapia combinada propicia altas taxas de controle da doença, preservação do aparelho de continência anal, com taxas de toxicidade aceitáveis e taxas de sobrevida sem necessidade de colostomia, e livres de doença satisfatórias.

O tratamento neo-adjuvante com terapia combinada foi mais utilizado no $2^{\circ}$ período. A terapia combinada conseguiu controlar a doença em $58 \%$ dos casos no $2^{\circ}$ período, evitando assim operações mutilantes. A perda de seguimento, que antigamente era de $57 \%$, foi de $16 \%$ no $2^{\circ}$ período.

Durante seguimento de tempo médio de 3,5 anos, houve $45 \%$ de sobrevida livre da doença no $2^{\circ}$ período. Dos pacientes seguidos ambulatorialmente, verificou-se mortalidade de $26 \%$. A alta taxa de mortalidade e o alto índice de recidiva indicam que tais lesões foram diagnosticadas tardiamente, com menor resposta ao tratamento QT e RT e cirurgia. Esses fatos estão relacionados com o nível sócio-econômico e cultural dos pacientes e por vezes pelo retardo do atendimento no sistema de saúde.

Dezoito por cento dos pacientes que apresentavam metástases à distância estavam vivos em 5 anos, comparados com $78 \%$ dos pacientes que apresentavam doença localizada. Doença em estágio avançado está relacionada a pior sobrevida, indicando que a detecção precoce pode melhorar a sobrevida dos pacientes com câncer anal ${ }^{12}$.

Dos pacientes seguidos ambulatorialmente, verificou-se mortalidade de $26 \%$. A alta taxa de mortalidade e o alto índice de recidiva indicam que tais lesões foram diagnosticadas tardiamente, com menor resposta ao tratamento QT e RT e cirurgia. Esses fatos estão relacionados com o nível sócio-econômico e cultural dos pacientes, e por vezes pelo retardo do atendimento no sistema de saúde.

\section{CONCLUSÕES}

1) No $1^{\circ}$ período (1979-1996, 17 anos de estudo) foram catalogados 23 casos, enquanto no $2^{\circ}$ período (1997-2004, 7 anos de estudo) foram registrados 27 casos, praticamente $60 \%$ de aumento no número de casos da doença diagnosticados em nosso serviço, confirmando o aumento desta patologia em todo o mundo. Este aumento pode ser atribuído, em parte, à epidemia HIV/SIDA e ao aumento dos casos de infecção pelo HPV. 
2) Carcinoma espinocelular permanece como neoplasia mais freqüente em ambos os períodos (2/3). O aumento nos casos de adenocarcinoma no $2^{\circ}$ período (1/4) reflete o aumento das neoplasias de reto baixo que invadem o canal anal.

3) Aumento significativo no uso da terapia combinada (Nigro) no $2^{\circ}$ período $(95 \%)$ reflete a tendência mundial para o tratamento padrão das neoplasias do canal anal; tal terapia propicia altas taxas de controle da doença, preservação do aparelho de continência anal, com taxas de toxicidade aceitáveis e taxas de sobrevida sem necessidade de colostomia, e livre de doenças satisfatórias. A terapia combinada conseguiu controlar a doença em $58 \%$ dos casos no $2^{\circ}$ período, evitando assim operações mutilantes (RAP).

\begin{abstract}
In total, 49 cases of anal tumor were studied, 23 cases from 1979-1996 and 26 cases from 1997-2004. From the cases studied, $49 \%$ were male and $51 \%$ were female patients. Patients that were submitted to radio and chemotherapy by the "nigro scheme" totalized $81.6 \% ; 12.2 \%$ presented metastasis, $36.7 \%$ lost follow up and $20.4 \%$ died. There was a reduction of the Miles surgery indication (30.8 \% vs $43.3 \%)$ and the follow up loss $(\mathbf{1 9 . 5} \%$ vs $56.5 \%)$. The mortality rate was a little higher $(23.1 \%$ vs $17.4 \%$ ). Radio and chemotherapy was used in $96 \%$ of patients, and promoted lesion remission in $57.5 \%$ of cases (confirmed by negative anal scar biopsy), avoiding the anal amputation surgery indication. Recent data indicate that $45 \%$ of patients (3.5 years of average follow up) are still with no recidivated tumor. The prevalence of high mortality rates and the diagnosis of high stage lesions are due to a delay of patients in search for a diagnosis. This might be due to a difficult access to a medical service and to the low social-economical level of our patients. These conditions make the diagnosis and treatment of anal tumors difficult, impairing its prognosis.
\end{abstract}

Key words: Anal tumor, casuistic, epidemiology.

\section{REFERÊNCIAS}

1. Nilsson PJ, Svensson C, Goldman S, Ljungqvist O, Glimelius B. Epidermoid anal cancer: a review of a population-based series of 308 consecutive patients treated according to prospective protocols. Int J Radiat Oncol Biol Phys. 2005 Jan 1;61(1):92-102.

2. Maggard MA, Beanes SR, Ko CY. Anal canal cancer: a population-based reappraisal. Dis Colon Rectum. 2003 Nov;46(11):1517-23. .

3. Melbye M, Rabkin C, Frisch M, Biggar RJ. Changing patterns of anal cancer incidence in the United States, 1940-1989. Am J Epidemiol. 1994 Apr 15;139(8):772-80.

4. Cress RD, Holly EA. Incidence of anal cancer in California: increased incidence among men in San Francisco, 1973-1999. Prev Med. 2003 May;36(5):555-60.

5. Frisch M. On the etiology of anal squamous carcinoma. Dan Med Bull. 2002 Aug;49(3):194-209.

6. Wong S, Gibbs P, Chao M, Jones I, McLaughlin S, Tjandra J, Faragher I, Green M. Carcinoma of the anal canal: a local experience and review of the literature. ANZ J Surg. 2004 Jul;74(7):541-6.

7. Touboul E, Moureau-Zabotto L, Lerouge D, Pene F, DeniaudAlexandre E, Tiret E, Sezeur A, Houry S, Gallot D, Parc R, Schlienger M, Laugier A. Radiotherapy of carcinomas of the anal canal. Tenon Hospital experience. Cancer Radiother. 2003 Nov;7 Suppl 1:91s-99s.
8. Clark MA, Hartley A, Geh JI. Cancer of the anal canal. Lancet Oncol. 2004 Mar;5(3):149-57.

9. Dem A, Kasse AA, Diop M, Toure P. Cancers of the anus: 32 cases. Dakar Med. 2000;45(1):74-6.

10. Sideris L, Lasser P, Elias D, Pocard M. Salvage surgery for anal canal carcinomas. Bull Cancer. 2004 Nov;91(11):839-44.

11. Einstein MH, Kadish AS. Anogenital neoplasia in AIDS. Curr Opin Oncol. 2004 Sep;16(5):455-62.

12. Johnson LG, Madeleine MM, Newcomer LM, Schwartz SM, Daling JR. Anal cancer incidence and survival: the surveillance, epidemiology, and end results experience, 1973-2000. Cancer. 2004 Jul 15;101(2):281-8.

13. Sobhani I, Walker F, Roudot-Thoraval F, Abramowitz L, Johanet H, Henin D, Delchier JC, Soule JC. Anal carcinoma: incidence and effect of cumulative infections. AIDS. $2004 \mathrm{Jul}$ 23;18(11):1561-9.

14. Frisch M. On the etiology of anal squamous carcinoma. Dan Med Bull. 2002 Aug;49(3):194-209.

15. Levi F, Te VC, Randimbison L, La Vecchia C. Incidence of anal carcinoma in Vaud, Switzerland, 1979-2001. Eur J Cancer Prev. 2004 Jun;13(3):213-5.

16. Akbari RP, Paty PB, Guillem JG, Weiser MR, Temple LK, Minsky BD, Saltz L, Wong WD. Oncologic outcomes of salvage surgery for epidermoid carcinoma of the anus initially managed with combined modality therapy. Dis Colon Rectum. 2004 Jul;47(7):1136-44. Epub 2004 May 28. 
17. Berry JM, Palefsky JM, Welton ML. Anal cancer and its precursors in HIV-positive patients: perspectives and management. Surg Oncol Clin N Am. 2004 Apr;13(2):355-73.

18. Skibber J, Rodriguez-Bigas MA, Gordon PH. Surgical considerations in anal cancer. Surg Oncol Clin N Am. 2004 Apr;13(2):321-38.

19. Eng C, Abbruzzese J, Minsky BD. Chemotherapy and radiation of anal canal cancer: the first approach. Surg Oncol Clin N Am. 2004 Apr;13(2):309-20, viii.

20. Khatri VP, Chopra S. Clinical presentation, imaging, and staging of anal cancer. Surg Oncol Clin N Am. 2004 Apr;13(2):295308.

21. Welton ML, Sharkey FE, Kahlenberg MS. The etiology and epidemiology of anal cancer. Surg Oncol Clin N Am. 2004 Apr;13(2):263-75.

22. Rousseau DL Jr, Petrelli NJ, Kahlenberg MS. Overview of anal cancer for the surgeon. Surg Oncol Clin N Am. 2004 Apr;13(2):249-62.

23. Borovac N. Carcinoma of the anus, rectum and colon. Med Arh. 2003;57(1 Suppl 2):87-9.

24. Mistrangelo M, Mobiglia A, Mussa B, Bello M, Pelosi E, Goss M, Bosso MC, Moro F, Sandrucci S. The sentinel node in anal carcinoma. Tumori. 2002 May-Jun;88(3):S51-2.

25. Newlin HE, Zlotecki RA, Morris CG, Hochwald SN, Riggs CE, Mendenhall WM. Squamous cell carcinoma of the anal margin. J Surg Oncol. 2004 May 1;86(2):55-62; discussion 63.

26. Abbasakoor F, Srivastava V, Swarnkar K, Stephenson BM. Implantation anal metastases after out-patient treatment of haemorrhoids. Ann R Coll Surg Engl. 2004 Jan;86(1):38-9.

27. Clark MA, Hartley A, Geh JI. Cancer of the anal canal. Lancet Oncol. 2004 Mar;5(3):149-57.

28. Bai YK, Cao WL, Gao JD, Liang J, Shao YF. Surgical salvage therapy of anal cancer. World J Gastroenterol. 2004 Feb 1;10(3):424-6.

29. Tseng HF, Morgenstern H, Mack TM, Peters RK. Risk factors for anal cancer: results of a population-based case-control study. Cancer Causes Control. 2003 Nov;14(9):837-46.

30. Chauveinc L, Buthaud X, Falcou MC, Mosseri V, De la Rochefordiere A, Pierga JY, Girodet J, Salmon RJ. Anal canal cancer treatment: practical limitations of routine prescription of concurrent chemotherapy and radiotherapy. Br J Cancer. 2003 Dec 1;89(11):2057-61.

31. Shirouzu K, Ogata Y, Araki Y, Kishimoto Y, Sato Y. A new ultimate anus-preserving operation for extremely low rectal cancer and for anal canal cancer. Tech Coloproctol. 2003 Oct;7(3):203-6.

32. Kocakova I, Kocak I, Vyzula R, Perkova H. Concomitant preoperative chemoradiotherapy in the treatment of anal carcinoma. Cas Lek Cesk. 2003;142 Suppl 1:36-9.

33. Deniaud-Alexandre E, Touboul E, Tiret E, Sezeur A, Houry S, Gallot D, Parc R, Huang R, Qu SH, Pene F, Schlienger M. Epidermoid carcinomas of the anal canal treated with definitive radiation therapy in a series of 305 patients. Cancer Radiother. 2003 Aug;7(4):237-53.

34. Deniaud-Alexandre E, Touboul E, Tiret E, Sezeur A, Houry S, Gallot D, Parc R, Huang R, Qu SH, Huart J, Pene F, Schlienger M. Results of definitive irradiation in a series of 305 epidermoid carcinomas of the anal canal. Int J Radiat Oncol Biol Phys. 2003 Aug 1;56(5):1259-73.

35. Klencke BJ, Palefsky JM. Anal cancer: an HIV-associated cancer. Hematol Oncol Clin North Am. 2003 Jun;17(3):85972.

36. Ortholan C, Francois E, Gerard JP. Preneoplastic anal lesions and anal canal carcinoma. Bull Cancer. 2003 May;90(5):40511.

37. Graf R, Wust P, Hildebrandt B, Gogler H, Ullrich R, Herrmann R, Riess H, Felix R. Impact of overall treatment time on local control of anal cancer treated with radiochemotherapy. Oncology. 2003;65(1):14-22.

38. Hill J, Meadows H, Haboubi N, Talbot IC, Northover JM. Pathological staging of epidermoid anal carcinoma for the new era. Colorectal Dis. 2003 May;5(3):206-13.

39. Serkies K, Bednaruk-Mlynski E, Dziadziuszko R, Jassem J. Conservative treatment for carcinoma of the anus-a report of 35 patients. Neoplasma. 2003;50(2):152-8.

40. Bendell JC, Ryan DP. Current perspectives on anal cancer. Oncology (Huntingt). 2003 Apr;17(4):492-7, 502-3; discussion 503, 507-9.

41. Lee DH, Kim I, Song HH, Jung JY, Kim DY, Lee KW, Kim TY, Heo DS, Bang YJ, Ha SW, Park JG, Kim NK. Induction chemotherapy followed by radiotherapy in the treatment of anal cancer. Oncol Rep. 2003 Jan-Feb;10 (1):101-4.

42. Belliere A, Chapet O, Coquard R, Romestaing P, Ardiet JM, Gerard JP. Brachytherapy in carcinomas of anal canal and rectum: techniques and results. Cancer Radiother. 2003 Feb;7(1):24-32.

43. Hung A, Crane C, Delclos M, Ballo M, Ajani J, Lin E, Feig B, Skibber J, Janjan N. Cisplatin-based combined modality therapy for anal carcinoma: a wider therapeutic index. Cancer. 2003 Mar 1;97(5):1195-202.

44. Radosevic-Jelic Lj, Stojanovic S, Durbaba M, Josifovski J, Krivokapic Z. Carcinoma of the anal canal—results of radical radiotherapy. Acta Chir Iugos1. 2002;49(2):79-83.

45. Michek J, Cierny M, Kubacak J, Sutory M, Kozumplik L, Vrastyak J. Tumors of the rectum and anus. Bratisl Lek Listy. 2002;103(11):411-3.

46. Fenger C. Prognostic factors in anal carcinoma. Pathology. 2002 Dec;34(6):573-8.

47. Moore HG, Guillem JG. Anal neoplasms. Surg Clin North Am. 2002 Dec; 82 (6):1233-51.

48. Wilt JA, George JM, Chung RT, Cook RL, O'Malley JE. Anal cancer in Alaska; a retrospective study of incidence, treatment, and outcomes. Alaska Med. 2002 Jul-Sep;44(3):56-9, 62.

49. Nigro ND, Seydel HG, Considine B, Vaitkevicius VK, Leichman L, Kinzie JJ. Combined preoperative radiation and 
chemotherapy for squamous cell carcinoma of the anal canal. Cancer (1983) 51: 1826-1829.

50. Keighley MRB, Williams NS. Surgery of the Anus, Rectum and Colon. W.B Sauders Company Ltd (1998), 1038-1072.

51. Miles WE. The radical abdomino-perineal operation for cancer of the rectum and of the pelvic colon. Br Med J 2 (1910); 941942.

52. Melbye M, Rabkin C, Frisch M, Biggar R. Changing patterns of anal cancer incidence in the United States, 1940-1989. Am J Epidemiol 139 (1994), pp. 772-780.

53. Goldman, SBG, Nilsson B, Pahlman L. Incidence of anal epidermoid carcinoma in Sweden 1970-1984. Acta Chir Scand 155 (1989), pp 191-197.

54. Frisch M, Melbye M, Moller H. Trends in incidence of anal cancer in Cenmark. BJM, 306 (1993), pp. 419-422.

55. Melbye M, Cote T, Kessler L, Gall M, Biggar R. High incidence of anal cancer amorg AIDS patients. Lancet 343 (1994), pp. 636-639.

56. Holly E, Whittemore A, Aston D, Ahn D, Nickoloff B, Kristiansen J. Anal cancer incidence: genital warts, anal fissure or fistula, hemorrhoids and smoking. J Natl Cancer Inst 81 (1989), pp. 1726-1731.

57. Critchlow C, Surawicz C, Holmes K, Kuypers J, Daling J, Hawes S, Goldbaum G, Sayer J, Hurt C, Dunphy C, Kiviat N. Prospective study of high grade anal squamous intraepithelial neoplasia in a cohort of homosexual men: influence of HIV infection, immunosupression and human pappillomavirus infection. AIDS 9 (1995), pp 1255-1262.

58. Whiteford MH, Stevens KR, Oh S, Deveney KE. The evolving treatment of anal cancer: how are we doing? Arch. Surg. 2001; 136: 886-91.

59. Ryan DP, Compton CC, Mayer RJ. Carcinoma of the anal canal. N. Engl. J. Med. 2000; 342: 792-800
60. Nigro N, Vaitkevicius VK, Considine B. Combined therapy for cancer of the anal canal: a preliminary report. Dis. Colon Rectum 1974; 17: 354-6.

61. Flam M, John M, Pajak TF et al. Role of mitomycin in combination with fluorouracil and radiotherapy, and of salvage chemoradiation in the definitive nonsurgical treatment of epidermoid carcinoma of the anal canal: results of a phase III randomised intergroup study. J. Clin. Oncol. 1996; 14: 2527-9.

62. Nilsson PJ, Svensson C, Goldman S, Glimelius B. Salvage abdominoperineal resection in anal epidermoid cancer. Br. J. Surg. 2002; 89: 1425-9

63. UKCCCR Anal Cancer Trial Working Party. Epidermoid anal cancer: results from the UKCCCR randomised trial of radiotherapy alone versus radiotherapy, 5-fluorouracil, and mitomycin C. Lancet 1996; 348: 1049-54

64. Bartelink H, Roelofsen F, Eschwege F et al. Concomitant radiotherapy and chemotherapy is superior to radiotherapy alone in the treatment of locally advanced anal cancer: results of a phase III randomized trial of the European Organization for Research and Treatment of Cancer Radiotherapy and Gastrointestinal Cooperative Groups. J. Clin. Oncol. 1997; 15: 2040-9.

65. Esiashvili N, Landry J, Matthews R et al. Carcinoma of the anus: strategies in management. Oncologist 2002; 7: 188-99.

\section{Endereço para correspondência: OMAR FERES}

Departamento de Cirurgia e Anatomia, Disciplina da Faculdade de Medicina de Ribeirão Preto, Universidade de São Paulo. CEP 14090-900 Ribeirão Preto/SP, Brasil.

Fone: 55-16-6022509

E-mail: feresomar@netsite.com.bre marlenelucio@yahoo.com.br 